\title{
The perceived causal relations between sensory reactivity differences and anxiety symptoms in autistic adults
}

Running Head: Sensory and anxiety: Perceived causal relations

\section{Authors}

*Isabelle Verhulst

*Keren MacLennana (corresponding author: kerenmaclennan@gmail.com)

Anthony Haffeya

Teresa Tavassolia

* Verhulst, I. and MacLennan, K. are co-first authors on this paper.

a The University of Reading, Earley Gate, Whiteknights Road, Reading, Berkshire, RG6 6ES, UK

๖Royal Holloway, University of London, Egham Hill, Egham, TW20 0EX, UK

Conflicts of interest: None

\section{Acknowledgements and author contributions}

Special thanks to all the autistic adults who participated in our research. Thanks to Dan Brady of the University of Reading for his assistance with the coding of the survey. Thanks to MQ, Autistica, the Centre for Autism at the University of Reading, and Autism Berkshire for helping with recruitment. I. Verhulst conducted the research design, programming of the survey, recruitment, data collection, data analysis, and dissertation writing. K. MacLennan conducted additional data analysis and wrote this manuscript. A. Haffey provided statistical support to this project. T. Tavassoli conducted the research design and supervised the project.

Funding: This work was co-supported by MQ: Transforming Mental Health and Autistica (grant number MQF17/6).

Preprint: This manuscript has been deposited to the PsyArXiv preprint server (https://psyarxiv.com/), https://doi.org/10.31234/osf.io/2nhbv 


\begin{abstract}
Background: Rates of anxiety are inordinately high in autistic adults. Sensory reactivity differences, such as hyperreactivity (e.g., strong reactions to sound), hyporeactivity (e.g., no, or slower reactions to pain), and seeking (e.g., fascination with spinning objects) are a diagnostic criterion of autism and have been linked with anxiety. Understanding how individuals perceive these to be causally related can impact assessment and treatment of anxiety. Therefore, we examined the perceived causal relations between sensory reactivity differences and anxiety in autistic adults.

Method: 246 autistic adults aged $18-76$ years took part in an online study. They completed self-report assessments of sensory reactivity differences, and anxiety, followed by the perceived causal relations scale; indicating if they perceived their sensory reactivity differences to be more of a cause or an effect of their anxiety symptoms.

Results: We found sensory reactivity hyperreactivity, hyporeactivity, and seeking to be significantly correlated with anxiety. Furthermore, we found total sensory hyperreactivity, and visual, auditory, and olfactory hyperreactivity, to be perceived as significantly more of a cause of anxiety than an effect, and total sensory seeking, and tactile and vestibular seeking, to be perceived as significantly more of an effect of anxiety than a cause.

Conclusion: Future individualized approaches to treating anxiety in autistic individuals may benefit from differentiating between potential sensory causes of anxiety (e.g., hypersensitivities) vs. potential sensory effects of anxiety (e.g., sensory seeking behaviours).
\end{abstract}

\title{
Community brief
}

Why is this an important issue?

Autistic people are more likely to experience anxiety compared to the general population. Sensory reactivity differences, such as hyperreactivity (e.g., strong reactivity to sounds), hyporeactivity (e.g., not noticing touch), or seeking (e.g., being fascinated by spinning objects), are common in autistic individuals, and may be a risk factor for anxiety. But existing anxiety treatments are not always effective for autistic people. Understanding how autistic people feel their anxiety and sensory reactivity differences are causally linked could be important to help clinicians understand the challenges that should be prioritised in anxiety treatments for autistic people.

What was the purpose of this study?

In this study we aimed to examine sensory reactivity differences and anxiety symptoms in autistic adults, and ask them if they perceive their anxiety symptoms to be more of a cause or an effect of their sensory reactivity differences. 
In our study we measured sensory reactivity differences and anxiety symptoms using online surveys. For the sensory reactivity differences and anxiety symptoms that each individual reported to be present, they were then asked how much they felt each anxiety symptom was a cause of their sensory reactivity differences, and how much they felt each sensory reactivity difference was a cause of their anxiety symptoms. This study included 246 autistic adults aged 18-76 years.

\section{What were the results of the study?}

Our results showed that the autistic individuals felt that their sensory hyperreactivity, including hyperreactivity related to vision, hearing, and scent, is more of a cause than an effect of anxiety. But also, that their sensory seeking, especially touch and balance related seeking is more of an effect than a cause of anxiety.

What do these findings add to what was already known?

Although sensory hyperreactivity has been shown before to be a cause of anxiety for autistic individuals, our study was the first to suggest that anxiety may influence sensory seeking, which is something that can be tested in future research studies.

What are potential weaknesses in the study?

We did not ask participants about co-occurring conditions, such as if they have additional diagnoses related to intellectual disability or attention deficit hyperreactivity disorder (ADHD), which means there may be important differences between individuals that we did not examine. Also, we did not measure key symptoms associated with wider anxiety conditions and autism-related anxiety symptoms, such as social fears unrelated to negative self-assessment, which would be important for clinical understanding.

How will these findings help autistic adults now or in the future?

Understanding how people perceive their traits and symptoms to be causally related can impact how effective anxiety treatments are for individuals. So, our findings can importantly help inform clinical approaches to the treatment of anxiety for autistic individuals.

Key words: Autism, anxiety, sensory, perceived causal relations, adults 


\section{Introduction}

Autism spectrum conditions (ASC) are neurodevelopmental conditions with diagnostic criteria relating to social interaction and communication differences, and restricted and repetitive behaviours and interests $\left(\right.$ RRBs $\left.^{1}\right)$. Autistic ${ }^{*}$ adults are at greater risk of experiencing clinical anxiety compared to the general population, with lifetime prevalence estimates suggesting it affects $42 \%$ of autistic adults. ${ }^{4}$ Prevalence rates for specific anxiety conditions vary across studies, but generalised anxiety disorder (GAD), separation anxiety, and social anxiety disorder are all commonly diagnosed in autistic children and adults. ${ }^{5-7}$ Anxiety has a significant impact on daily living and quality of life for autistic individuals, ${ }^{8,9}$ which is thought to be greater than the impact of being autistic. ${ }^{8}$ Accurate assessment and identification of anxiety in autistic individuals is important for access to appropriate interventions. ${ }^{5}$ However, there is currently a lack of understanding of anxiety presentations in autistic individuals. ${ }^{10-13}$ Therefore, it is important to understand more about autism-specific risk factors, such as sensory reactivity differences.

Sensory reactivity differences, a criterion under the RRBs domain, have been suggested as a risk factor for anxiety in autism. ${ }^{14}$ Sensory reactivity differences include sensory hyperreactivity (i.e., strong reactions to sensory input such as sounds), hyporeactivity (i.e., no, or slower reactions to sensory input such as pain), and seeking (i.e., fascination or fixation with sensory input such as watching spinning objects). Individuals often experience varying patterns of differences across a range of contexts and modalities, such as vision, hearing, and touch. ${ }^{15-17}$ Sensory reactivity differences are commonly experienced by approximately $94 \%$ of autistic adults, ${ }^{18}$ but are also often present more generally in neurodivergent individuals, such as with the condition clinically known as Attention Deficit Hyperactivity Disorder (ADHD). ${ }^{19}$ Although some sensory input can be experienced as enjoyable, other input can be extremely distressing and sometimes painful for individuals. ${ }^{20,21}$ This can cause individuals to feel overwhelmed in their daily lives and is thought to affect long term physical and mental health outcomes, including anxiety. ${ }^{20}$ Subsequently, sensory reactivity differences and anxiety have been associated with wider impacts, such as reduced engagement with activities, public spaces (e.g., public transport), and workplaces for autistic individuals. ${ }^{9,22,23}$

Sensory hyperreactivity is commonly experienced across a range of modalities (e.g., visual, auditory), and is associated with inordinate distress and feelings of overwhelm. ${ }^{20}$ In research, it has frequently been linked to anxiety in autistic children and adults. ${ }^{21,24-28}$ Although it has more recently been understood that autistic individuals can experience distinct autism-related anxiety symptomology, such as social fears unrelated to negative self-appraisal, sensory hyperreactivity has been found to predict traditional anxiety symptomology aligning with the DSM, ${ }^{1}$ rather than distinct anxiety symptomology. ${ }^{29}$ For instance, previous research has found sensory hyperreactivity to be related to symptomology associated with GAD, separation anxiety, social anxiety, and specific phobia. ${ }^{24,27-30}$

\footnotetext{
* The language used in this article reflects the preferred terms of the autistic community ${ }^{2}$ and importantly supports the reduction of stigma. ${ }^{3}$
} 
Researchers have suggested three theoretical models conceptualising the possible link between sensory hyperreactivity and anxiety. ${ }^{31}$ The Primary Anxiety Model proposes anxiety to be a cause of sensory hyperreactivity, as individuals may be more likely to be conditioned to attend and overreact to sensory input due to characteristics of anxiety (e.g., hypervigilance, hyperarousal, attentional bias, and avoidance). Secondly, the Primary SOR (sensory over-responsivity) Model proposes sensory hyperreactivity to be a cause for anxiety, as aversive sensory input can be conditioned, either towards a specific stimulus or across contexts due to the uncontrollability or unpredictability of stimuli. Thirdly, it is proposed there may be a non-causal link between sensory hyperreactivity and anxiety, but the association is due to an additional factor, for instance similarities in amygdala activation or diagnostic overlap. In support of the primary hyperreactivity model, longitudinal work in autistic toddlers has found sensory hyperreactivity to be an early unidirectional predictor of anxiety in autistic individuals. ${ }^{25}$ Additionally, there is some evidence to support that the link may be due to common overactivation in the amygdala and hippocampus. ${ }^{32}$ However, more research is needed to unpick the causal association between sensory hyperreactivity and anxiety.

Relatively little research has examined whether sensory hyporeactivity and seeking relate to anxiety in autistic individuals. Research that has included sensory hyporeactivity has inconsistently found evidence of a significant relationship to anxiety. Some previous work has found greater sensory hyporeactivity to be related to greater anxiety in autistic adults and children. ${ }^{26,33}$ However, our previous work in autistic children found sensory hyporeactivity to be related to lower anxiety, including symptomology relating to social anxiety when controlling for broader autistic traits. ${ }^{27}$ Alternatively, research in autistic children has suggested that sensory hyporeactivity may instead be indirectly related to anxiety through RRBs. ${ }^{34}$ Whereas for sensory seeking, quantitative research in autistic children and non-autistic adults has primarily not found a significant link with anxiety. ${ }^{16,27,28,35}$ However, a study with neurodivergent young people, which included autistic children and teenagers, has shown a link between sensory seeking and anxiety, specifically GAD. ${ }^{36}$ Qualitative research in autistic adults has indicated that sensory seeking can be a soothing or calming strategy in times of stress. ${ }^{20,37,38}$ Moreover, researchers have suggested that sensory seeking may be indirectly linked to anxiety as a strategy to regulate arousal, either seeking out sensory input in response to hyporeactivity, or interlinked with RRBs to soothe in response to sensory hyperreactivity. ${ }^{16}$ Although sensory hyporeactivity and seeking are prominent in adulthood, research examining their association with anxiety is vastly lacking, with no known studies yet to examine the relationship between sensory seeking and anxiety in autistic adults.

Although it is important to elucidate the causal associations between sensory reactivity differences and anxiety, examining how autistic individuals perceive these to be causally related can also importantly improve the effectiveness of anxiety treatments. Although individuals often perceive their behaviours and clinical symptoms to be causally related, clinical assessments primarily assess co-occurring symptomology in isolation. ${ }^{39}$ Assessing perceived causal relations (PCR) of co-occurring psychological behaviours/symptoms at an individual case level could be important for individualising clinical assessments, providing information and context of the challenges that should be addressed and at what stage in treatment. ${ }^{40,41}$ The PCR scale is a rating scale used to understand the cognitive perception of 
causal relationship between two or more symptoms. ${ }^{39,40}$ PCR provides a more reflective account of the perceived causal relationship than, for example, inferring causal associations from statistically modelling questionnaire data. It can provide detailed insight at idiographic level, or aggregated to group level, and is therefore a beneficial approach to inform the development of targeted treatments. ${ }^{39}$ PCR has previously been used in autism research to analyse relationships between Obsessive Compulsive Disorder and autistic repetitive behaviours. ${ }^{42}$ It is especially relevant for treatment approaches to anxiety and other mental health conditions in autistic individuals due to the heterogenous nature of this population. ${ }^{43}$ Furthermore, there has been a push to increase stakeholder involvement in autism research, which has previously not been common practice. Using a method such as PCR brings the perspectives of autistic individuals into research. Thus, findings are more likely to reflect and represent lived experiences and consequently improve outcomes for autistic people. ${ }^{44}$

Understanding how sensory reactivity differences are associated with anxiety, and perceptions of the causal relationship, has important implications for treatments approaches that are effective and suitable for autistic adults. Therefore, we conducted an online study that was the first known to examine the correlational and perceived causal relations between sensory reactivity differences (hyperreactivity, hyporeactivity, and seeking, across domains, including visual, auditory, tactile, interoception, vestibular, gustatory, and olfactory), and anxiety in autistic adults. Firstly, we aimed to investigate if sensory reactivity differences are associated with anxiety in autistic adults, examining sensory seeking for the first time. Secondly, we aimed to understand if autistic adults perceive their sensory reactivity differences to be more of a cause or an effect of their anxiety symptoms, using the novel PCR scale approach.

\section{Methods}

\section{Participants}

We recruited 282 autistic adults for this online study. Inclusion followed a two-step process: firstly, participants were included who self-reported that they had an official autism diagnosis; and secondly participants had to score above the cut-off $(\geq 6)$ on the Autism Spectrum Quotient - 10 (AQ-10). ${ }^{45}$ Therefore, 246 participants were included in the final analysis (aged 18-76 years, $M=41.9, S D=13.7$, 143 female, 21 trans female, and 82 male). We did not record specific data on race and ethnicity, socioeconomic status, and educational attainment levels. We recruited via the Centre for Autism Database at the University of Reading, the Autism Research Centre, and UK autistic adults' charities Facebook pages. Participants provided informed consent to take part. Ethical approval was granted prior to the commencement of this study by the University of Reading Ethics Committee.

Measures

Sensory Processing Scale Inventory (SPSI)

The SPSI ${ }^{46}$ is a self-report questionnaire assessment of sensory reactivity differences for use in children and adults. The SPSI assesses sensory hyperreactivity, hyporeactivity, and seeking across a range of 
domains (auditory, visual, tactile, olfactory, gustatory, vestibular, and interoception). It is usually scored dichotomously $(1=$ yes, $0=$ no), however, to capture a wider range scores and more sensitive differences between participants, Likert-scale scoring was used, in-line with the Sensory Profile scoring (1 - 5; almost always, frequently, occasionally, seldom, almost never). ${ }^{47}$ The measure has good internal reliability (hyperreactivity $=0.89$; hyporeactivity $=0.88$; sensory seeking $=0.93$ ), and discriminant validity (medium to large effect sizes; .56-.1.53). ${ }^{46,48}$

\section{Screen for Adult Anxiety Related Disorders (SCAARED)}

The SCAARED ${ }^{49}$ is a self-report questionnaire screening assessment of DSM anxiety disorders for use in adults. The SCAARED assesses total anxiety symptoms, as well as specific anxiety disorders including GAD, social anxiety, separation anxiety, and is scored $0-2$ using a Likert-scale (not true or hardly ever true, somewhat true or sometimes true, very true or often true). It has good to excellent internal consistency $(0.86-0.94)$, and discriminant validity. ${ }^{49}$ Although the SCAARED has not specifically been validated for use with autistic people, the scale is adapted from the child version (Screen for Child Anxiety Related Emotion Disorders ${ }^{50}$ ), which has been found to have comparable psychometric properties across autistic and non-autistic samples. ${ }^{51}$ Questionnaires of anxiety validated in the general population are commonly used in autism research due to the lack of population specific measures.

\section{Perceived Causal Relations (PCR) scaling}

We used the PCR scaling method ${ }^{39}$ to examine the cognitive perception of the causal relationships between sensory reactivity differences and anxiety symptoms. In the PCR scale we used the items of the SPSI and SCAARED questionnaires, asking participants to which extent they perceive their sensory reactivity differences (hyperreactivity, hyporeactivity, and seeking in each domain, e.g., auditory hyperreactivity) to be a cause of anxiety (generalised anxiety, separation anxiety, and social anxiety), and to which extent they perceive their anxiety to be a cause of their sensory reactivity differences.

In the case that participants endorsed items relating to sensory reactivity differences as well as anxiety, they were asked to "Rate on a 0-10 scale ( $0=$ 'not at all', $5=$ 'moderate cause', $10=$ 'strong cause'), to which extent you think your [X sensory reactivity difference] causes your [Y anxiety symptom]?" and "Rate on a $0-10$ scale ( $0=$ 'not at all', $5=$ 'moderate cause', $10=$ 'strong cause') to which extent you think your [Y anxiety symptom] causes your [X sensory reactivity difference]?" The number of items in the PCR scale was unique for each participant depending on the amount of sensory reactivity differences and anxiety symptoms that were present. A sensory reactivity difference on the SPSI was included if participants rated the item as 'frequently' or 'almost always', and an anxiety symptom was included if participants rated the item as 'very true or often true'. If participants endorsed all sensory reactivity differences and anxiety symptomologies, the maximum number of PCR items would be 60 (20 SPSI subscales $\times 3$ SCAARED subscales). Missing PCR items (because of SPSI or SCAARED items not being endorsed) were treated as missing values at an individual case level. From the PCR items, we calculated cause and effect composite scores for total sensory reactivity differences 
(hyperreactivity, hyporeactivity, and seeking) but also sensory reactivity differences by domain (visual, auditory, tactile, interopection, vestibular, gustatory, and olfactory).

\section{Procedure}

We used Gorilla experiment builder (www.gorilla.sc) to develop and host this study. ${ }^{52}$ Participants accessed the study via a direct link to the study's Gorilla webpage. Participants were then able to read the study information and provided informed consent electronically, before completing the demographic information and the standardised questionnaires (AQ-10, SPSI, and SCAARED; the SPSI and SCAARED were presented in a counterbalanced order). Participants then completed the PCR scale, informed by their answers to the SPSI and SCAARED items.

\section{Analysis}

We conducted statistical analysis using JASP, ${ }^{53}$ a statistical program based on R, and SPSS. ${ }^{54}$ Following normality checks, we firstly conducted a Spearman's bivariate correlation analysis to examine the associations between sensory reactivity (hyperreactivity, hyporeactivity, and seeking), and anxiety, as well as the covariates (autistic traits and age). Secondly, we examined if anxiety was perceived to be more the cause or the effect of sensory reactivity differences (hyperreactivity, hyporeactivity, and seeking, overall and in each domain). For the PCR analysis we conducted a series of paired-samples $\mathrm{t}$-tests to test if the mean cause was significantly different from the mean effect. ${ }^{39}$ As items were variable at a case level, depending on if an item was endorsed or not by a participant, each analysis varied in sample size. A significant correlation between constructs, but not a significant difference between cause and effect, may indicate the perception of a non-causal association.

\section{Community involvement}

We conducted this research study with an autistic researcher as part of the core research team. Additionally, the research questions for this study align with the research priorities as identified by stakeholders in association with Autistica and the James Lind Alliance. ${ }^{55}$

\section{Results}

\section{Descriptive statistics}

Shapiro-Wilk test of normality indicated that not all the variables were normally distributed $(p<.05)$ (see Table 1 for descriptive statistics). Based on scores from the SCAARED, 85\% scored above the cut-off for GAD, $68 \%$ scored above the cut-off for separation anxiety disorder, and $85 \%$ scored above the cut-off for social anxiety disorder, indicating clinically elevated symptoms indicative of these anxiety disorders. 
Table 1

Descriptive statistics for sensory reactivity differences (SPSI), anxiety (SCAARED), autism traits (AQ-10), and age.

\begin{tabular}{lllll}
\hline & Range & Mean & SD & SE \\
\hline Hyperreactivity & $15-73$ & 51.21 & 10.82 & .69 \\
Hyporeactivity & $12-46$ & 25.76 & 6.37 & .41 \\
Seeking & $10-46$ & 24.32 & 7.38 & .47 \\
Total anxiety & $0-54$ & 33.15 & 11.27 & .72 \\
Autistic traits & $6-10$ & 8.48 & 1.28 & .08 \\
Age & $18-76$ & 41.86 & 13.70 & .87 \\
\hline
\end{tabular}

\section{Correlation analysis}

We conducted a Spearman's bivariate correlation analysis, with a Bonferroni corrected alpha level of $p$ $\leq .017(.05 / 3)$, to examine the relationships between sensory reactivity and anxiety (see Table 2 for correlation results and Supplementary Item 1 for scatterplot matrix). We found that sensory hyperreactivity was significantly correlated with total anxiety $\left(r_{\mathrm{s}}=.383, p<.001\right)$, sensory hyporeactivity was significantly correlated with total anxiety $\left(r_{\mathrm{s}}=.157, p=.014\right)$ and sensory seeking was significantly correlated with total anxiety $\left(r_{\mathrm{s}}=.295, p<.001\right)$. We did not find age or autistic traits to be significant covariates after Bonferroni correction (7 comparisons: adjusted $p>.006$ ). However, pre-correction, we found age to be significantly correlated with sensory seeking $\left(r_{\mathrm{s}}=-.171, p=.007\right)$.

Table 2

Spearman's correlations between sensory reactivity differences (SPSI), anxiety (SCAARED), autism traits (AQ-10), and age.

\begin{tabular}{llllll}
\hline & Hyperreactivity & Hyporeactivity & Seeking & Autistic traits & Age \\
\hline Total anxiety & $\mathbf{. 3 8 3 ^ { * * * } ( . 0 0 0 )}$ & $\mathbf{. 1 5 7 ^ { * } ( . 0 1 4 )}$ & $\mathbf{. 2 9 5 ^ { * * * } ( . 0 0 0 )}$ & $.027(.674)$ & $-.063(.325)$ \\
Autistic traits & $.122(.056)$ & $.121(.057)$ & $.038(.551)$ & - & - \\
Age & $-.054(.403)$ & $-.123(.054)$ & $\mathbf{- . 1 7 1 ( . 0 0 7 )}$ & $.095(.135)$ & - \\
\hline
\end{tabular}

*. Correlation is significant at the Bonferroni corrected $p \leq 0.016(.05 / 3)$ level (2-tailed)

**. Correlation is significant at the Bonferroni corrected $p \leq 0.008(.05 / 6)$ level (2-tailed)

$* * *$. Correlation is significant at the $p \leq 0.001$ level (2-tailed)

Correlations significant prior to Bonferroni correction are indicated in bold without being flagged.

\section{Perceived causal relations between sensory reactivity differences and anxiety}

We conducted a series of paired-samples t-tests (Wilcoxon signed-rank) to examine if sensory reactivity differences were perceived to be significantly more of a cause or an effect of anxiety. We applied a Bonferroni corrected significance level of $p=.017$ (.05/3 tests) to control for multiple tests. See Table 3 for summary of these results.

We found that sensory hyperreactivity was perceived to be a significantly greater cause of anxiety (PCR hyper $\rightarrow$ anx $; M=4.62, S D=2.20)$, than an effect of anxiety ( $P C R_{a n x \rightarrow h y p e r ;} M=4.29, S D=2.41$ ), $Z(238)=15864.5, p=.001, r_{r b}=.248$. Whereas, we found that sensory seeking was perceived to be $a$ significantly greater effect of anxiety $\left(P C R_{a n x} \rightarrow\right.$ seek$\left.; M=3.60, S D=2.60\right)$, than a cause of anxiety $\left(P_{\text {CReek } \rightarrow \text { anx }} ; M=3.14, S D=2.50\right), Z(215)=6142.5, p=.008, r_{r b}=-.229$.

We then conducted a series of paired-samples t-tests (Wilcoxon signed-rank) to examine if sensory reactivity differences in specific domains were significantly perceived to be more of a cause or an 
effect of anxiety. We applied a Bonferroni corrected significance level of $p=.0025$ (.05/20 tests) to control for multiple tests. See Table 3 for summary of these results.

We found that auditory hyperreactivity was perceived to be a significantly greater cause of anxiety ( $\left.P C R_{\text {audhyper } \rightarrow \text { anx }} ; M=5.73, S D=2.51\right)$, than an effect of anxiety $\left(P C R_{a n x \rightarrow \text { audhyper }} ; M=5.07, S D=\right.$ 2.83), $\mathrm{Z}(229)=11229.5, \mathrm{p}<.001, \mathrm{r}_{\mathrm{rb}}=.291$, olfactory hyperreactivity was perceived to be a significantly greater cause of anxiety ( $\left.P C R_{\text {offhyper } \rightarrow \text { anx }}, M=4.28, S D=2.55\right)$, than an effect of anxiety ( $\left.P C R_{a n x \rightarrow o l f h y p e r ; ~} M=3.67, S D=2.76\right), Z(183)=7405.5, p=.001, r_{r b}=.308$, and visual hyperreactivity was perceived to be a significantly greater cause of anxiety $\left(P C R_{\text {vishyper } \rightarrow \text { anx }} ; M=4.14, S D=2.14\right)$, than an effect of anxiety ( $\left.P C R_{a n x \rightarrow v i s h y p e r ;} M=4.75, S D=2.77\right), Z(205)=8080.5, p=.002, r_{r b}=.287$. Whereas, we found that tactile seeking was perceived to be a significantly greater effect of anxiety ( $\left.P C R_{\text {anx } \rightarrow \text { tactseek; }} ; M=4.86, S D=2.88\right)$, than a cause of anxiety $\left(P C R_{\text {tactseek } \rightarrow \text { anx }} ; M=2.91, S D=2.75\right)$, $Z(88)=31.5, p<.001, r r b=-.789$, and vestibular seeking was perceived to be a significantly greater effect of anxiety $\left(P C R_{a n x \rightarrow \text { vestseek}} ; M=3.40, S D=3.10\right)$, than a cause of anxiety $\left(P C R_{\text {vesttseek } \rightarrow \text { anx }} ; M=\right.$ $2.51, \mathrm{SD}=2.59), \mathrm{Z}(40)=31.5, \mathrm{p}<.001, \mathrm{r}_{\mathrm{rb}}=-.806$.

Table 3

Paired-samples t-test results summary for Perceived Causal Relations between sensory reactivity differences and anxiety. Significant $t$-tests indicate that sensory is perceived to be more a cause of anxiety ( $P C R_{\text {sensory } \rightarrow \text { anxiety) }}$ ) than anxiety is perceived to be a cause of sensory (PCRanxiety $\rightarrow$ sensory) or vice versa.

\begin{tabular}{|c|c|c|c|c|c|c|c|c|c|c|}
\hline & \multirow[b]{2}{*}{$\mathbf{N}$} & \multicolumn{2}{|c|}{$\mathrm{PCR}_{\text {sensory } \rightarrow \text { anxiety }}$} & \multicolumn{2}{|c|}{$\mathrm{PCR}_{\text {anxiety } \rightarrow \text { sensory }}$} & \multirow[b]{2}{*}{$\mathbf{Z}$} & \multirow[b]{2}{*}{$\mathbf{p}$} & \multirow[b]{2}{*}{$\mathrm{r}_{\mathrm{rb}}$} & \multirow[b]{2}{*}{$1-\beta$} & \multirow[b]{2}{*}{ Sig PCR } \\
\hline & & M & sd & M & sd & & & & & \\
\hline Hyperreactivity & 238 & 4.62 & 2.20 & 4.29 & 2.41 & 15864.5 & $.001^{*}$ & .25 & .84 & $\mathrm{PCR}_{\text {sensory }} \rightarrow$ anxiety \\
\hline Visual & 205 & 5.14 & 2.73 & 4.75 & 2.77 & 8080.5 & $.002^{* *}$ & .29 & .65 & $\mathrm{PCR}_{\text {sensory } \rightarrow \text { anxiety }}$ \\
\hline Auditory & 229 & 5.73 & 2.51 & 5.07 & 2.83 & 11229.5 & $<.001^{* *}$ & .29 & .97 & $\mathrm{PCR}_{\text {sensory } \rightarrow \text { anxiety }}$ \\
\hline Tactile & 206 & 4.19 & 2.58 & 4.39 & 2.76 & 5622.0 & .164 & -.13 & .25 & - \\
\hline Interoception & 94 & 4.91 & 2.55 & 4.87 & 2.56 & 1641.5 & .013 & .013 & .05 & - \\
\hline Vestibular & 167 & 4.44 & 2.69 & 4.22 & 2.75 & 4004.5 & .092 & .18 & .38 & - \\
\hline Gustatory & 131 & 3.62 & 2.78 & 3.15 & 2.96 & 3144.5 & .006 & .32 & .63 & - \\
\hline Olfactory & 183 & 4.28 & 2.55 & 3.67 & 2.76 & 7405.5 & $.001^{* *}$ & .31 & .93 & $\mathrm{PCR}_{\text {sensory } \rightarrow \text { anxiety }}$ \\
\hline Hyporeactivity & 217 & 3.41 & 2.48 & 3.71 & 2.46 & 7071.5 & .099 & -.14 & .56 & - \\
\hline Visual & 46 & 4.05 & 2.76 & 4.48 & 2.78 & 233.5 & .41 & -.17 & .20 & - \\
\hline Auditory & 156 & 3.41 & 2.72 & 3.22 & 2.66 & 3940.0 & .18 & .14 & .13 & - \\
\hline Tactile & 113 & 3.26 & 2.78 & 3.84 & 2.80 & 1025.0 & .004 & -.37 & .76 & - \\
\hline Interoception & 94 & 4.05 & 3.03 & 4.67 & 3.21 & 1094.5 & .057 & -.25 & .48 & - \\
\hline Vestibular & 96 & 3.19 & 2.52 & 3.52 & 2.76 & 1066.6 & .40 & -.12 & .27 & - \\
\hline Gustatory & 41 & 3.73 & 2.70 & 3.73 & 2.81 & 224.0 & .90 & .03 & .05 & - \\
\hline Olfactory & 25 & 3.07 & 2.62 & 2.71 & 2.65 & 71.5 & .53 & .19 & .11 & - \\
\hline Seeking & 215 & 3.14 & 2.50 & 3.60 & 2.60 & 6142.5 & $.008^{*}$ & -.23 & .87 & PCR anxiety $\rightarrow$ sensory \\
\hline Visual & 105 & 3.86 & 2.74 & 4.31 & 2.87 & 1340.5 & .095 & -.21 & .35 & - \\
\hline Auditory & 77 & 4.39 & 2.88 & 4.29 & 2.66 & 787.5 & .701 & .06 & .07 & - \\
\hline Tactile & 88 & 2.91 & 2.75 & 4.86 & 2.88 & 233.0 & $<.001^{* *}$ & -.79 & .99 & $\mathrm{PCR}_{\text {anxiety } \rightarrow \text { sensory }}$ \\
\hline Vestibular & 40 & 2.51 & 2.59 & 3.40 & 3.10 & 31.5 & $<.001^{* *}$ & -.81 & .94 & $\mathrm{PCR}_{\text {anxiety }} \rightarrow$ sensory \\
\hline Gustatory & 168 & 2.96 & 2.71 & 2.91 & 2.81 & 3939.0 & .633 & .05 & .06 & - \\
\hline Olfactory & 72 & 3.51 & 2.85 & 4.15 & 2.86 & 534.0 & .073 & -.28 & .46 & - \\
\hline
\end{tabular}

${ }^{*}$. Correlation is significant at the Bonferroni corrected $p \leq 0.017(0.05 / 3)$ level (2-tailed)

**. Correlation is significant at the $p \leq 0.0025(0.05 / 20)$ level (2-tailed)

Correlations significant prior to Bonferroni correction are indicated in bold without being flagged. 


\section{Discussion}

Our study is the first to advance our understanding of the perceived causal relationships between sensory reactivity differences and anxiety in autistic adults. Firstly, we found a significant correlational relationship between sensory hyperreactivity and anxiety and sensory hyporeactivity and anxiety, in line with our previous work and that of others. ${ }^{25-28,34}$ However, we also found sensory seeking to be significantly correlated with anxiety, the first known to examine this relationship in autistic adults. Furthermore, for the first time we found that autistic adults perceive sensory hyperreactivity, notably visual, auditory, and olfactory hyperreactivity, to be more of a cause of their anxiety symptoms than an effect, and that they perceive sensory seeking, notably tactile and vestibular seeking, to be more of an effect of their anxiety symptoms than a cause. However, it is important to note that due to the limited samples sizes in some of the domain-specific PCR analyses, it may be that significance was not achieved due to lack of power (e.g., gustatory hyporeactivity and seeking).

Research in autistic children and adults, including the present results, have consistently found a significant link between sensory hyperreactivity and anxiety. ${ }^{25-28,34}$ Furthermore, as we found autistic adults perceive sensory hyperreactivity to be more of a cause than an effect of their anxiety symptoms, this aligns with longitudinal work that has found sensory hyperreactivity to be a unidirectional predictor of anxiety. ${ }^{25}$ Hence, our results provide further support for the Primary SOR Model. ${ }^{31}$ Sensory hyperreactivity may cause anxiety due to autism related difficulties with the unpredictability of aversive sensory input, which may lead to anxiety developing through context conditioning from increased hypervigilance, negative bias, and avoidance of generalised contexts. ${ }^{31,56}$ Interestingly, our results indicate that hyperreactivity in the visual, auditory, and olfactory domains is perceived to be more of a cause of anxiety symptoms than an effect. Visual and auditory input is more commonly associated with sensory hyperreactivity in autistic individuals than with hyporeactivity and seeking. ${ }^{20,57}$ Visual, auditory and olfactory hyperreactivity, such as difficulties with bright or flickering lights, sudden loud sounds, and intense scents, have been commonly reported by autistic individuals and are associated with distress, overwhelm, and anxious responses. ${ }^{20}$ Therefore, sensory input associated with these domains may be more likely to condition anxious responses in individuals compared to other domain input. Overall, our findings suggest that supporting challenges with sensory hyperreactivity could be an important first step in treatment approaches for anxiety in autistic adults. There is some evidence to suggest that adapted Cognitive Behavioural Therapy, supporting self-understanding of sensory reactivity differences and the development of coping strategies, could be used to target anxiety arising from sensory hyperreactivity. ${ }^{58}$ Due to the complex and individualistic nature of sensory experiences, ${ }^{20}$ it may be that clinical services could support individuals to identify and develop personal strategies to help them navigate and reduce the impact of hyperreactivity.

As for sensory seeking, our study was the first known to examine its associations with anxiety in autistic adults. Previous work in autistic children ${ }^{16,27,28}$ has primarily not found a significant association between sensory seeking and anxiety. However, a study with neurodivergent young people, including autistic children and teenagers, found sensory seeking to be associated with anxiety, specifically GAD. ${ }^{36}$ Our study builds on existing research to suggest that autistic adults perceive it to be an outcome of anxiety. 
This may suggest sensory seeking is a regulatory strategy and reflect findings from existing qualitative work in this population, which has shown that sensory seeking behaviours can be experienced as soothing or calming in times of stress. ${ }^{20,37,38}$ The domain results indicate that autistic adults perceive tactile and vestibular seeking to be more of an effect of their anxiety symptoms than a cause. Autistic adult commonly seek out tactile experiences, such certain soft or fluffy textures, and feeling warm or cold surfaces on their skin. ${ }^{20}$ As for vestibular seeking, autistic adults engaging in stimming behaviours, such as spinning or rocking, to self-regulate and calm when feeling overwhelmed or anxious. ${ }^{59,60}$ Selfsoothing behaviours have been linked to the release of oxytocin, important for wellbeing and stress reduction, and the anti-stress effects of oxytocin release have been suggested to be acutely strong in response to 'low intensity' stimulation of the skin. ${ }^{61}$ Thus, seeking out enjoyable tactile input and vestibular behaviours may be important self-soothing strategies that can be supported and developed at a later stage in therapeutic approaches for anxiety in autistic adults.

Regarding sensory hyporeactivity, although we found a significant correlation with anxiety, we did not find a significant causal association, suggesting autistic individuals do not perceive it to be causally implicated in their anxiety symptoms. Research has commonly been inconsistent in regard to sensory hyporeactivity, with some finding it to be related to anxiety in autistic adults and children, ${ }^{26,27,33,62}$ whilst others have not. ${ }^{28,34}$ Sensory hyporeactivity has been suggested to be experienced after periods of hyperreactivity, where individuals may inversely 'shutdown' from sensory input. ${ }^{63,64}$ Therefore, sensory hyporeactivity may be implicated in anxiety indirectly due to its association with hyperreactivity.

\section{Limitations}

Due to the heterogeneous nature of autism, individuals present with varying strengths and difficulties as well as co-occurring conditions. ${ }^{43}$ Therefore, it could have been informative if we collected additional demographic information, such as co-occurring diagnoses (e.g., Intellectual Disability or ADHD), to ascertain if there were any key sub-group differences. Future research should examine the influence of other individual factors, as well as aiming to replicate these findings in more specific subgroups of autistic adults, as this may have an important impact on clinical approaches.

Although GAD, separation anxiety, and social anxiety can all co-occur in autistic individuals, our study did not examine additional common anxiety symptomology, such as specific phobia, or examine anxiety symptoms that are distinct to autistic individuals. ${ }^{5,6}$ Although sensory reactivity differences have been found to predict traditional anxiety symptomology, there is growing evidence to suggest that anxiety presentations differ in autistic individuals compared to the general population, such as social fears that are not related to negative self-appraisal. ${ }^{29}$ Research has yet to examine associations between sensory reactivity differences and distinct autism-related anxiety symptomology in autistic adults. Since the conception of this study, there has been the development of an anxiety questionnaire for use with autistic adults, ${ }^{65}$ which we could now use in future research to further elucidate the links between sensory reactivity differences and anxiety symptomology in autistic individuals. 


\section{Conclusion}

Our study found sensory hyperreactivity, hyporeactivity, and seeking to be correlated with anxiety in autistic adults. Furthermore, we found that autistic adults perceive sensory hyperreactivity to be more of a cause than an effect of their anxiety symptoms, but sensory seeking to be more of an effect than a cause of their anxiety symptoms. Our results have implications for future research directions, informing hypotheses for objective longitudinal research designs. Additionally, our findings have clinical implications for the progression of individualised treatment approaches for anxiety in autistic individuals. 


\section{References}

1. DSM-5 American Psychiatric Association. Diagnostic and Statistical Manual of Mental Disorders 5 (DSM-5). Arlington: American Psychiatric Publishing; 2013.

2. Kenny L, Hattersley C, Molins B, Buckley C, Povey C, Pellicano E. Which terms should be used to describe autism? Perspectives from the UK autism community. Autism. 2016;20(4):442-462. doi: $10.1177 / 1362361315588200$

3. Gernsbacher MA. Editorial Perspective: The use of person-first language in scholarly writing may accentuate stigma. Journal of Child Psychology and Psychiatry. 2017;58(7):859-861. doi:10.1111/jcpp.12706

4. Hollocks MJ, Lerh JW, Magiati I, Meiser-Stedman R, Brugha TS. Anxiety and depression in adults with autism spectrum disorder: a systematic review and meta-analysis. Psychol Med. 2019;49(4):559-572. doi:10.1017/S0033291718002283

5. Kerns CM, Winder-Patel B, losif AM, et al. Clinically Significant Anxiety in Children with Autism Spectrum Disorder and Varied Intellectual Functioning. Journal of Clinical Child \& Adolescent Psychology. Published online January 23, 2020:1-16. doi:10.1080/15374416.2019.1703712

6. Nimmo-Smith V, Heuvelman H, Dalman C, et al. Anxiety Disorders in Adults with Autism Spectrum Disorder: A Population-Based Study. J Autism Dev Disord. 2020;50(1):308-318. doi:10.1007/s10803-019-04234-3

7. Simonoff E, Pickles A, Charman T, Chandler S, Loucas T, Baird G. Psychiatric Disorders in Children With Autism Spectrum Disorders: Prevalence, Comorbidity, and Associated Factors in a Population-Derived Sample. Journal of the American Academy of Child \& Adolescent Psychiatry. 2008;47(8):921-929. doi:10.1097/CHI.0b013e318179964f

8. Ozsivadjian A, Knott F, Magiati I. Parent and child perspectives on the nature of anxiety in children and young people with autism spectrum disorders: a focus group study. Autism. 2012;16(2):107-121. doi:10.1177/1362361311431703

9. Robertson AE, Stanfield AC, Watt J, et al. The experience and impact of anxiety in autistic adults: A thematic analysis. Research in Autism Spectrum Disorders. 2018;46:8-18. doi:10.1016/j.rasd.2017.11.006

10. Camm-Crosbie L, Bradley L, Shaw R, Baron-Cohen S, Cassidy S. 'People like me don't get support': Autistic adults' experiences of support and treatment for mental health difficulties, selfinjury and suicidality. Autism. 2019;23(6):1431-1441. doi:10.1177/1362361318816053

11. Crane L, Adams F, Harper G, Welch J, Pellicano E. 'Something needs to change': Mental health experiences of young autistic adults in England. Autism. 2019;23(2):477-493. doi:10.1177/1362361318757048

12. Kerns CM, Renno P, Kendall PC, Wood JJ, Storch EA. Anxiety Disorders Interview ScheduleAutism Addendum: Reliability and Validity in Children With Autism Spectrum Disorder. Journal of Clinical Child \& Adolescent Psychology. 2017;46(1):88-100. doi:10.1080/15374416.2016.1233501

13. Maddox BB, Crabbe S, Beidas RS, et al. "I wouldn't know where to start": Perspectives from clinicians, agency leaders, and autistic adults on improving community mental health services for autistic adults. Autism. 2020;24(4):919-930. doi:10.1177/1362361319882227

14. South M, Rodgers J. Sensory, Emotional and Cognitive Contributions to Anxiety in Autism Spectrum Disorders. Frontiers in Human Neuroscience. 2017;11.

doi:10.3389/fnhum.2017.00020 
15. Baranek GT, David FJ, Poe MD, Stone WL, Watson LR. Sensory Experiences Questionnaire: discriminating sensory features in young children with autism, developmental delays, and typical development: SEQ. Journal of Child Psychology and Psychiatry. 2006;47(6):591-601. doi:10.1111/j.1469-7610.2005.01546.x

16. Lidstone J, Uljarević M, Sullivan J, et al. Relations among restricted and repetitive behaviors, anxiety and sensory features in children with autism spectrum disorders. Research in Autism Spectrum Disorders. 2014;8(2):82-92. doi:10.1016/j.rasd.2013.10.001

17. Miller LJ, Anzalone ME, Lane SJ, Cermak SA, Osten ET. Concept evolution in sensory integration: a proposed nosology for diagnosis. Am J Occup Ther. 2007;61(2):135-140. doi:10.5014/ajot.61.2.135

18. Crane L, Goddard L, Pring L. Sensory processing in adults with autism spectrum disorders. Autism. 2009;13(3):215-228. doi:10.1177/1362361309103794

19. Miller LJ, Nielsen DM, Schoen SA. Attention deficit hyperactivity disorder and sensory modulation disorder: A comparison of behavior and physiology. Research in Developmental Disabilities. 2012;33(3):804-818. doi:10.1016/j.ridd.2011.12.005

20. MacLennan K, O'Brien S, Tavassoli T. In Our Own Words: The Complex Sensory Experiences of Autistic Adults. J Autism Dev Disord. Published online July 13, 2021. doi:10.1007/s10803021-05186-3

21. Uljarević M, Lane A, Kelly A, Leekam S. Sensory subtypes and anxiety in older children and adolescents with autism spectrum disorder: Sensory Subtypes and Anxiety in Autism. Autism Research. 2016;9(10):1073-1078. doi:10.1002/aur.1602

22. Amos GA, Byrne G, Chouinard PA, Godber T. Autism Traits, Sensory Over-Responsivity, Anxiety, and Stress: A Test of Explanatory Models. Journal of Autism and Developmental Disorders. 2019;49(1):98-112. doi:10.1007/s10803-018-3695-6

23. Parmar KR, Porter CS, Dickinson CM, Pelham J, Baimbridge P, Gowen E. Visual Sensory Experiences From the Viewpoint of Autistic Adults. Front Psychol. 2021;12:633037. doi:10.3389/fpsyg.2021.633037

24. Black KR, Stevenson RA, Segers M, et al. Linking Anxiety and Insistence on Sameness in Autistic Children: The Role of Sensory Hypersensitivity. Journal of Autism and Developmental Disorders. 2017;47(8):2459-2470. doi:10.1007/s10803-017-3161-x

25. Green SA, Ben-Sasson A, Soto TW, Carter AS. Anxiety and Sensory Over-Responsivity in Toddlers with Autism Spectrum Disorders: Bidirectional Effects Across Time. Journal of Autism and Developmental Disorders. 2012;42(6):1112-1119. doi:10.1007/s10803-011-1361-3

26. Hwang YI (Jane), Arnold S, Srasuebkul P, Trollor J. Understanding anxiety in adults on the autism spectrum: An investigation of its relationship with intolerance of uncertainty, sensory sensitivities and repetitive behaviours. Autism. Published online August 15, 2019:136236131986890. doi:10.1177/1362361319868907

27. MacLennan K, Roach L, Tavassoli T. The Relationship Between Sensory Reactivity Differences and Anxiety Subtypes in Autistic Children. Autism Research. 2020;13(5):785-795. doi:10.1002/aur.2259

28. MacLennan $\mathrm{K}$, Rossow $\mathrm{T}$, Tavassoli $\mathrm{T}$. The relationship between sensory reactivity, intolerance of uncertainty and anxiety subtypes in preschool-age autistic children. Autism. Published online May 24, 2021:136236132110161. doi:10.1177/13623613211016110

29. Kerns CM, Kendall PC, Berry L, et al. Traditional and Atypical Presentations of Anxiety in Youth with Autism Spectrum Disorder. Journal of Autism and Developmental Disorders.

2014;44(11):2851-2861. doi:10.1007/s10803-014-2141-7 
30. Bitsika V, Arnold WA, Sharpley CF. The Role of Sensory Features in Mediating Associations Between Autism Symptoms and Anxiety in Boys with Autism Spectrum Disorder. Journal of Autism and Developmental Disorders. Published online February 15, 2019. doi:10.1007/s10803019-03917-1

31. Green SA, Ben-Sasson A. Anxiety Disorders and Sensory Over-Responsivity in Children with Autism Spectrum Disorders: Is There a Causal Relationship? Journal of Autism and Developmental Disorders. 2010;40(12):1495-1504. doi:10.1007/s10803-010-1007-x

32. Green SA, Rudie JD, Colich NL, et al. Overreactive Brain Responses to Sensory Stimuli in Youth With Autism Spectrum Disorders. Journal of the American Academy of Child \& Adolescent Psychiatry. 2013;52(11):1158-1172. doi:10.1016/j.jaac.2013.08.004

33. Glod M, Riby DM, Rodgers J. Short report: Relationships between sensory processing, repetitive behaviors, anxiety, and intolerance of uncertainty in autism spectrum disorder and Williams syndrome. Autism Research. 2019;12(5):759-765. doi:10.1002/aur.2096

34. Wigham S, Rodgers J, South M, McConachie H, Freeston M. The Interplay Between Sensory Processing Abnormalities, Intolerance of Uncertainty, Anxiety and Restricted and Repetitive Behaviours in Autism Spectrum Disorder. Journal of Autism and Developmental Disorders. 2015;45(4):943-952. doi:10.1007/s10803-014-2248-x

35. Levit-Binnun N, Szepsenwol O, Stern-Ellran K, Engel-Yeger B. The relationship between sensory responsiveness profiles, attachment orientations, and anxiety symptoms: Sensory responsiveness, attachment, and anxiety. Australian Journal of Psychology. 2014;66(4):233240. doi:10.1111/ajpy.12064

36. Rossow T, Marco EJ, Cortica, San Rafael, California, USA, Gerdes M, Department of Neurology, University of California San Francisco, California, USA. The Relationship between Sensory Reactivity Differences and Mental Health Symptoms in Children with Neurodevelopmental Conditions and Their Neurotypical Peers. OBM Neurobiology. 2021;5(4):11. doi:10.21926/obm.neurobiol.2104110

37. Robertson AE, Simmons DR. The Sensory Experiences of Adults with Autism Spectrum Disorder: A Qualitative Analysis. Perception. 2015;44(5):569-586. doi:10.1068/p7833

38. Smith RS, Sharp J. Fascination and Isolation: A Grounded Theory Exploration of Unusual Sensory Experiences in Adults with Asperger Syndrome. Journal of Autism and Developmental Disorders. 2013;43(4):891-910. doi:10.1007/s10803-012-1633-6

39. Frewen PA, Allen SL, Lanius RA, Neufeld RWJ. Perceived Causal Relations: Novel Methodology for Assessing Client Attributions About Causal Associations Between Variables Including Symptoms and Functional Impairment. Assessment. 2012;19(4):480-493. doi:10.1177/1073191111418297

40. Frewen PA, Schmittmann VD, Bringmann LF, Borsboom D. Perceived causal relations between anxiety, posttraumatic stress and depression: extension to moderation, mediation, and network analysis. European Journal of Psychotraumatology. 2013;4(1):20656. doi:10.3402/ejpt.v4i0.20656

41. Haynes SN, Mumma GH, Pinson C. Idiographic assessment: Conceptual and psychometric foundations of individualized behavioral assessment. Clinical Psychology Review. 2009;29(2):179-191. doi:10.1016/j.cpr.2008.12.003

42. Ruzzano L, Borsboom D, Geurts HM. Repetitive Behaviors in Autism and ObsessiveCompulsive Disorder: New Perspectives from a Network Analysis. Journal of Autism and Developmental Disorders. 2015;45(1):192-202. doi:10.1007/s10803-014-2204-9

43. Vasa RA, Mazurek MO. An update on anxiety in youth with autism spectrum disorders: Current Opinion in Psychiatry. 2015;28(2):83-90. doi:10.1097/YCO.0000000000000133 
44. Fletcher-Watson S, Adams J, Brook K, et al. Making the future together: Shaping autism research through meaningful participation. Autism. Published online August 10, 2018:136236131878672. doi:10.1177/1362361318786721

45. Booth T, Murray AL, McKenzie K, Kuenssberg R, O'Donnell M, Burnett $\mathrm{H}$. Brief Report: An Evaluation of the AQ-10 as a Brief Screening Instrument for ASD in Adults. J Autism Dev Disord. 2013;43(12):2997-3000. doi:10.1007/s10803-013-1844-5

46. Schoen SA, Miller LJ, Sullivan J. The development and psychometric properties of the Sensory Processing Scale Inventory: A report measure of sensory modulation. Journal of Intellectual \& Developmental Disability. 2017;42(1):12-21. doi:10.3109/13668250.2016.1195490

47. Brown C, Tollefson N, Dunn W, Cromwell R, Filion D. The Adult Sensory Profile: Measuring Patterns of Sensory Processing. American Journal of Occupational Therapy. 2001;55(1):75-82. doi:10.5014/ajot.55.1.75

48. Schoen SA, Miller LJ, Green KE. Pilot Study of the Sensory Over-Responsivity Scales: Assessment and Inventory. American Journal of Occupational Therapy. 2008;62(4):393-406. doi:10.5014/ajot.62.4.393

49. Angulo M, Rooks BT, Gill M, et al. Psychometrics of the screen for adult anxiety related disorders (SCAARED)- A new scale for the assessment of DSM-5 anxiety disorders. Psychiatry Research. 2017;253:84-90. doi:10.1016/j.psychres.2017.02.034

50. Birmaher B, Khetarpal S, Brent D, et al. The Screen for Child Anxiety Related Emotional Disorders (SCARED): Scale Construction and Psychometric Characteristics. Journal of the American Academy of Child \& Adolescent Psychiatry. 1997;36(4):545-553. doi:10.1097/00004583-199704000-00018

51. van Steensel FJ, Deutschman AA, Bögels SM. Examining the Screen for Child Anxiety-Related Emotional Disorder-71 as an assessment tool for anxiety in children with high-functioning autism spectrum disorders. Autism. 2013;17(6):681-692. doi:10.1177/1362361312455875

52. Anwyl-Irvine AL, Massonnié J, Flitton A, Kirkham N, Evershed JK. Gorilla in our midst: An online behavioral experiment builder. Behav Res. 2020;52(1):388-407. doi:10.3758/s13428-01901237-x

53. JASP team. JASP (Version 0.14) [Computer Software].; 2020. https://jasp-stats.org/

54. IBM Corp. IBM SPSS Statistics for Mac.; 2020.

55. Cusack J, Sterry R. Your questions: Shaping future autism research. London: Autistica. Published online 2016.

56. Grillon C. Models and mechanisms of anxiety: evidence from startle studies. Psychopharmacology. 2008;199(3):421-437. doi:10.1007/s00213-007-1019-1

57. Tavassoli T, Bellesheim K, Siper PM, et al. Measuring Sensory Reactivity in Autism Spectrum Disorder: Application and Simplification of a Clinician-Administered Sensory Observation Scale. Journal of Autism and Developmental Disorders. 2016;46(1):287-293. doi:10.1007/s10803-0152578-3

58. Edgington L, Hill V, Pellicano E. The design and implementation of a CBT-based intervention for sensory processing difficulties in adolescents on the autism spectrum. Research in Developmental Disabilities. 2016;59:221-233. doi:10.1016/j.ridd.2016.09.004

59. Joyce C, Honey E, Leekam SR, Barrett SL, Rodgers J. Anxiety, Intolerance of Uncertainty and Restricted and Repetitive Behaviour: Insights Directly from Young People with ASD. J Autism Dev Disord. 2017;47(12):3789-3802. doi:10.1007/s10803-017-3027-2 
60. Kapp SK, Steward R, Crane L, et al. 'People should be allowed to do what they like': Autistic adults' views and experiences of stimming. Autism. 2019;23(7):1782-1792.

doi:10.1177/1362361319829628

61. Uvnäs-Moberg K, Handlin L, Petersson M. Self-soothing behaviors with particular reference to oxytocin release induced by non-noxious sensory stimulation. Front Psychol. 2015;5. doi:10.3389/fpsyg.2014.01529

62. Rossow $\mathrm{T}$, MacLennan $\mathrm{K}$, Tavassoli $\mathrm{T}$. The relationship between sensory reactivity differences and mental health symptoms in preschool-age autistic children. Autism Research. Published online May 4, 2021:aur.2525. doi:10.1002/aur.2525

63. Lane SJ. Sensory modulation. In: Bundy AC, Lane SJ, Murray EA, eds. Sensory Integration: Theory and Practice. 2nd ed. F. A. Davis.; 2002:101-122.

64. Liss M, Saulnier C, Fein D, Kinsbourne M. Sensory and attention abnormalities in autistic spectrum disorders. Autism. 2006;10(2):155-172. doi:10.1177/1362361306062021

65. Rodgers J, Farquhar K, Mason D, et al. Development and Initial Evaluation of the Anxiety Scale for Autism-Adults. Autism in Adulthood. 2020;2(1):24-33. doi:10.1089/aut.2019.0044 


\section{Supplementary Item 1}

Scatterplot matrix of the Spearman's correlations between sensory reactivity differences (SPSI), anxiety (SCAARED), autism traits (AQ-10), and age.
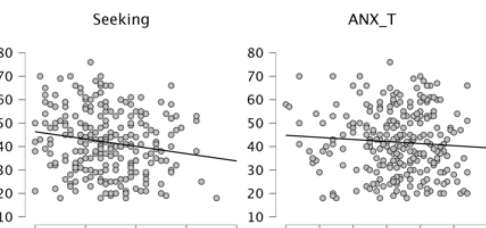

$10-$
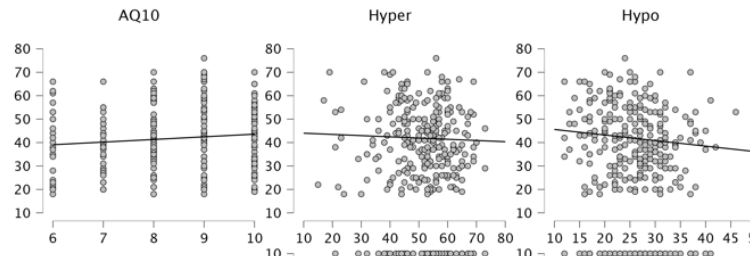

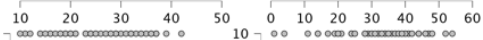

$\stackrel{9}{q}$
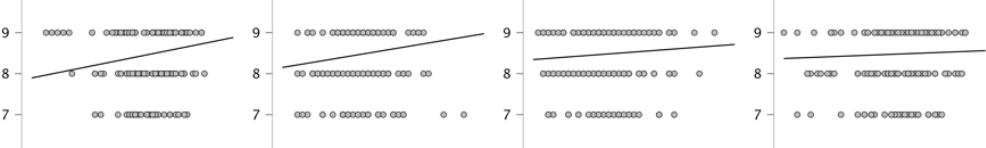

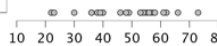
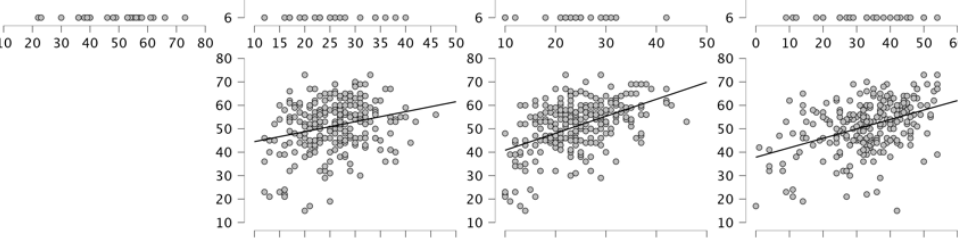

1015202530354045

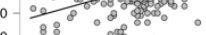

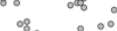

$\begin{array}{llllllll}0 & 10 & 20 & 30 & 40 & 50 & 60\end{array}$
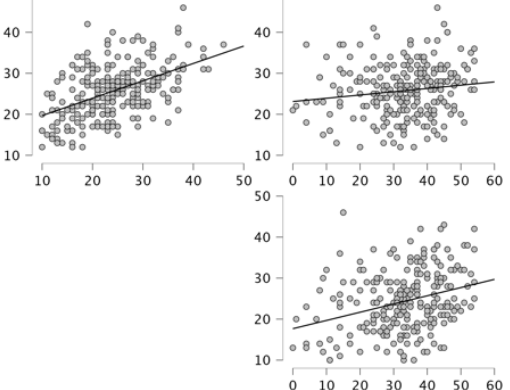